\title{
EI VOT: una opción atractiva en la administración del tratamiento de la tuberculosis
}

\author{
ROBERT HARTOG, MPH*
}

\author{
Video Observed Treatment, an attractive option for the administration \\ of tuberculosis therapy
}

VOT (Video Observed Treatment) is a video certificated self-administration of therapy and could be complementary to DOT (Directly Observed Treatment) for the administration of ambulatory tuberculosis treatment at Primary Health Centers. Reviewed international experience and evidence indicates that VOT improves treatment adherence, empowers patients, reduces health system costs and saves patient's transfer time to Therapy Centers. Given the high penetration of smartphones with videocall software in the Chilean population, the pre-requisites are provided to consider a VOT pilot research in Chile.

Key words: Tuberculosis; Treatment adherence and compliance, Video observed treatment; Directly observed treatment; Chile.

\section{Resumen}

El VOT (video observed treatment) es la autoadministración de la terapia certificada mediante registro de imágenes en video y podría constituir para algunos pacientes una alternativa complementaria al DOT (directly observed treatment) en la administración del tratamiento ambulatorio de la Tuberculosis en Centros de Salud Primarios. Existen evidencias internacionales en que la estrategia VOT mejora la adherencia al tratamiento, empodera a los pacientes, reduce los costos para pacientes y el sistema de salud y ahorra el tiempo dedicado por los pacientes al traslado a centros de terapia. La disponibilidad masiva de teléfonos portátiles con capacidad de trasmitir videos en la población de Chile podría permitir realizar una investigación piloto de VOT.

Palabras clave: Tuberculosis; Adherencia al tratamiento y cumplimiento; Video observed treatment; Directly observed treatment; Chile.

\section{EI DOT: Directly observed treatment}

La tuberculosis (TB) se ubica dentro de las 10 primeras causas de muerte a nivel mundial y requiere al menos 6 meses de tratamiento. La adherencia incompleta al tratamiento conlleva el riesgo de infecciosidad prolongada, así como el fracaso del tratamiento, la recaída y el desarrollo de las temidas resistencias a los medicamentos de primera línea. Por esto, completar el tratamiento a través del DOT es de máxima relevancia en la estrategia de tratamiento de la TB y su erradicación. La idea de utilizar el DOT se remonta a los años $50^{1}$, y Chile fue pionero en emplearlo desde los años 60². Desde 1993 la aplicación del DOT es recomendado por la Organización Mundial de la Salud $(\mathrm{OMS})^{3}$.

El DOT es un método de administración de medicamentos, en el cual un profesional de salud observa y registra diariamente cómo una persona toma cada dosis de medicamentos en forma presencial. El DOT en Chile es obligatorio y tiene una cobertura universal de $100 \%$ como indica la OMS. Con esta estrategia se pretende asegurar, que cada paciente tome todos los medicamentos en la forma prescrita.

En 2018 la tasa de incidencia de tuberculosis en Chile fue de 15,7 casos por 100.000 habitantes, correspondiendo a un total de 2.945 casos, y con tendencia levemente ascendente desde 2014.

* Sala Era, CESFAM Cristo Vive, Comuna de Recoleta. Santiago, Chile. 
Los principales condiciones de riesgo son adultos mayores $(20,3 \%)$, extranjeros $(22,8 \%)$, alcoholismo $(11,2 \%)$, drogadicción $(10,5 \%)$, co-infección VIH $(8,9 \%)$, diabetes $(8,2 \%)$, contactos $(5,4 \%)$, situación de calle $(4,2 \%)$, y otros $(10,7 \%)$. La tasa de extranjeros casi se duplicó entre los años 2016 a 2018, reflejando la creciente inmigración a Chile ${ }^{4}$.

En la evaluación de la cohorte de todos los casos nuevos de TB ingresados durante 2016 el Programa Nacional de Tuberculosis (PROCET) se constata no haber cumplido las metas propuestas, de lograr al menos un $90 \%$ de curación (llega a $78,9 \%$ ), tener menos de $5 \%$ de pérdidas en el seguimiento (llega a $6,6 \%$ ) y obtener una tasa de fallecimiento durante el tratamiento de menos de $3 \%$ (llega a 10,5\%) (PROCET 2018) $)^{5}$. Para mejorar los resultados, los autores concluyen, que es "necesario incorporar estrategias innovadoras que complementen a las tradicionales, tanto en implementación de tecnología, como en actividades para una mejor pesquisa y adherencia al tratamiento, con enfoque en los grupos vulnerables de la población".

A pesar de que el DOT ha sido una estrategia exitosa desde la perspectiva de salud pública, éste presenta dificultades para los pacientes por tiempo y costo de los traslados diarios. Al respecto, una revisión de literatura sobre adherencia al tratamiento de la TB (Munroe 2007) ${ }^{6}$, identificó los factores estructurales, personales, así como aquellos relacionados con los servicios de salud, que son determinantes para la adherencia de los pacientes al tratamiento. El estudio, entre otros, señaló que los pacientes pueden percibir el DOT como una pérdida de tiempo y como vergonzoso tener que ingerir los medicamentos bajo supervisión. Además, puede existir un conflicto entre cumplir con las obligaciones laborales o acudir al centro de salud. Para mejorar la adherencia, los autores recomiendan minimizar los costos relacionados con visitas a los centros de salud y aumentar la opción del paciente de elegir el tipo de apoyo que necesita.

\section{EI VOT: Video observed treatment}

El VOT es parecido al DOT, sólo que el paciente envía una grabación de la ingesta de los medicamentos al profesional de salud para su registro. El VOT puede ser síncrono, cuando un profesional observa la ingesta de los medicamentos mediante la transmisión directa de un video, o asíncrono, cuando éstas imágenes son grabadas y enviadas para su registro ulterior. El
VOT asíncrono tiene la ventaja, que el paciente puede tomar los medicamentos a su conveniencia.

El desarrollo tecnológico ha sido el motor del VOT. Desde la aparición de los primeros videophones al final de los años '90 surgió la idea de utilizarlos para el DOT. La gran mayoría de los estudios desde $2001^{7}$ en adelante, eran de interés pragmático: investigaron la factibilidad, la aceptabilidad y los costos del VOT. Todos indicaron, que el VOT era factible, generalmente preferido por los pacientes, y por lo menos igual de eficiente que el $\mathrm{DOT}^{8-11}$, si no superior.

Un ensayo prospectivo con 390 pacientes realizado entre septiembre 2013 y septiembre 2014 en Nueva York (Chuck 2016) ${ }^{12}$, encontró adherencia similar entre pacientes con DOT (329 pacientes, cumplimento $91 \%$ ) y el grupo con VOT (61 pacientes, cumplimiento 95\%). Los autores destacaron el uso mucho más eficiente de los recursos del servicio. Otros ensayos que medían los costos del VOT, reportaron ahorros hasta 2.240 dólares (USA) por caso tratado ${ }^{13-17}$, principalmente por eliminación de gastos asociados a visitas a domicilio.

Todos los estudios revisados destacan la aceptación positiva por parte de los pacientes, los que en general recomiendan este método por sobre el DOT. Los pacientes también perciben, que el VOT respeta su autonomía y sus preferencias individuales.

El VOT ya fue adoptado en muchos países desde 2010 en adelante, impulsado por la creciente disponibilidad de smartphones y el acceso a internet. Entre ellos países de ingresos altos, medianos y bajos, como EE.UU, Reino Unido, India, Kazakhstan, Australia, Bielorrusia, Kenya y Vietnam entre otros.

Una encuesta nacional del año 2015 a diferentes programas contra la tuberculosis en EE.UU, encontró que $47(42 \%)$ de los 113 programas que respondieron, ya estaban usando el VOT (en el estudio lo llaman $e D O T$ ) y un $36 \%$ planeaba implementarlo durante 2016 (Macaraig 2018) ${ }^{18}$. Otro estudio que realizó 56 entrevistas a personal de salud que trabajan con VOT en diferentes servicios en California (Ritter 2017) ${ }^{19}$, observó que existía un fuerte interés en utilizar el VOT, que las preocupaciones iniciales se reducían y que los trabajadores encontraron que los beneficios superaron sus expectativas.

Otra revisión sobre la literatura del VOT (Garfein 2019) ${ }^{20}$, basada en 22 publicaciones entre los años 2001 a 2018 concluye, que la evidencia acumulada muestra que el VOT es factible, aceptable y logra una adherencia al tratamiento comparable o mejor que el DOT, y a un costo inferior. Los 
autores del estudio señalaron, que para precisar la efectividad del VOT y las mejores prácticas para su implementación, resulta necesario realizar investigación adicional.

En el año 2015, la OMS y la European Respiratory Society, determinaron, que el VOT respondía positivamente al pilar "Atención y Prevención integradas y centradas en el paciente" (el primero de los 3 pilares de la "Estrategia Fin a la TB") ${ }^{21}$. En abril de 2015 la OMS estableció la Global Task Force on Digital Health ${ }^{22}$ para promover la integración de instrumentos de salud digital a los programas nacionales en su estrategia de eliminar la tuberculosis ${ }^{23}$. Esto incluía entre otros el VOT.

Desde 2017 la OMS respalda explícitamente el VOT como alternativa al DOT, incluyendo la implementación del VOT en sus recomendaciones para los Programas Nacionales de Control de la $\mathrm{TB}^{24}$, siempre cuando la capacidad tecnológica sea suficiente. Se trata de una recomendación condicional, dado que todavía no existía la evidencia científica suficiente sobre la efectividad del VOT. En consecuencia, la misma publicación nombra entre las prioridades para investigación, el estudio del uso del VOT en países de ingresos medianos y bajos, para mejorar la adherencia al tratamiento de la TB.

\section{Los estudios aleatorizados y controlados so- bre el VOT}

Posteriormente, se publicaron dos estudios que comparan directamente la efectividad del VOT con el DOT en un ensayo aleatorizado y controlado, es decir con un alto rigor metodológico.

Uno de estos estudios, se realizó en Moldavia (Ravenscroft 2020) ${ }^{25}$, a 197 pacientes, que mostró una adherencia al tratamiento significativamente superior en el grupo VOT frente al grupo DOT. Sin embargo, puesto que el tiempo de observación fue de tan solo 14 días, la validez del mismo parece limitada.

El otro estudio aleatorizado y controlado sobre el VOT, fue realizado entre 2014 y 2016 a 226 pacientes en Inglaterra (A. Story, 2019) ${ }^{26}$. El ensayo clínico comparó la efectividad del VOT versus el DOT durante 6 meses de tratamiento, proporcionándole a los pacientes del grupo VOT un smartphone con plan. Del total de estos pacientes, un 53\% estaba en situación de calle y tenía antecedentes de drogadicción, de uso de alcohol o problemas psiquiátricos. Al cabo de 2 meses el grupo con VOT logró $>80 \%$ de cumplimiento del tratamiento versus $31 \%$ de los pacientes con DOT. Este resultado fue influen- ciado por el abandono del tratamiento dentro de la primera semana de $54 \%$ de los pacientes del grupo DOT, y solamente $11 \%$ en el grupo VOT. Los autores concluyen, que en el caso del VOT, el tratamiento se hace mucho más aceptable para los pacientes cuando la oferta es más confortable, flexible, y menos invasiva. Además, destaca que en el grupo del VOT tomaban 7 dosis por semana en la fase diaria del tratamiento, mientras que en el DOT solo 5.

Los otros resultados del mismo estudio demuestran, que incluso en pacientes considerados como difíciles, con alta probabilidad de abandonar el tratamiento antes de terminarlo, el VOT puede funcionar incluso mejor que el DOT. En efecto, en ambos grupos que no abandonaron el tratamiento, el grupo VOT cumplió con el $83 \%$ de las dosis previstas a los 6 meses, versus el $61 \%$ del grupo DOT, mostrando una mejor adherencia al tratamiento.

Por otra parte, se midieron los tiempos destinados por los profesionales de salud a cada paciente al día: en promedio el DOT a domicilio utilizó $56 \mathrm{~min}$, el DOT en el servicio, $15 \mathrm{~min}$, mientras que el VOT, sólo 3,2 min. Para los pacientes, se calculó un promedio de 29 min para el DOT, y 1,8 min para el VOT.

Los investigadores observaron, además, que aun cuando no había contacto directo entre el paciente y el profesional de salud a cargo del tratamiento, no se afectaba la identificación de efectos secundarios por parte de estos últimos.

Los autores concluyen, que el VOT probablemente va a ser preferible sobre el DOT para un grupo grande de pacientes en muchas situaciones sociales distintas, procurando una opción más aceptable, más efectiva y más económica.

Basándose en los resultados de este último estudio, el "Find and Treat Service" del Reino Unido, que atiende los pacientes con TB en situación de calle, empezó a utilizar el VOT de forma rutinaria. En la actualidad, la mayoría de sus pacientes en Londres lo están utilizando ${ }^{27}$.

\section{Discusión}

El VOT ofrece una gran oportunidad para facilitar y mejorar la adherencia del paciente con TB a su tratamiento, reduciendo significativamente sus tiempos y costos de traslado y en especial en situaciones de aislamiento geográfico.

El VOT permite además continuar el tratamiento en días festivos o durante viajes cortos. Incluso en la primera fase del tratamiento, la fase diaria, en la cual el paciente toma los me- 
dicamentos de lunes a viernes, se podría incluir los sábados y domingos, tal como se hace con pacientes hospitalizados, y así acortar la duración de esta fase inicial del tratamiento de 10 semanas a 7 semanas.

En la actualidad, varios mecanismos de transmisión de información encriptan los mensajes, como por ejemplo, whatsapp, lo que garantizaría la protección de los contenidos enviados. Además, resulta el envío sin costo adicional, si el paciente posee un smartphone y tiene un plan con acceso a internet, los cuales generalmente permiten navegación libre en redes sociales. Al respecto, hay que tomar en cuenta que para el año 2017, según datos de la SUBTEL (Subsecretaría de Comunicaciones de Chile), el 87\% de los hogares chilenos tenía acceso a internet, entre los servicios fijos y móviles contratados ${ }^{28}$, con una tendencia ascendente. Con lo anterior, se puede asumir, que la gran mayoría de los pacientes tiene acceso a internet.

El VOT, además, permite transmitir rápidamente al personal de salud a través del mismo medio de mensajería o aplicación, dudas que presenten los pacientes sobre el tratamiento o posibles reacciones adversas a los medicamentos. Es una preocupación importante, ya que el DOT también sirve para pesquisar efectos secundarios. Sin embargo, los estudios revisados no muestran reducción de notificación de reacciones adver$\operatorname{sas}^{26}$. Además no hay que olvidar que igualmente se produce un contacto bisemanal con la TENS (técnico en enfermería de nivel superior) del programa y mensualmente con el médico.

En la situación actual de cuarentenas repetitivas, el VOT podría ser una ayuda para evitar que los pacientes tengan que ir a su centro de salud si otras posibilidades están limitadas (se puede recurrir a una terapia domiciliaria por personal de salud capacitado como alternativa actualmente aceptada).

Las normas técnicas del PROCET (MINSAL $2014)^{29}$, contenidas en un decreto con fuerza de Ley, exigen observar en forma directa como el paciente ingiere sus medicamentos. En su Articulo 95 dice: La administración del tratamiento será de preferencia ambulatoria, lo más cercano al domicilio o lugar de trabajo del paciente, $y$ siempre estrictamente supervisada, es decir, directamente observada por personal de salud capacitado y, en casos excepcionales, por otras personas capacitadas a quienes se encomiende esta responsabilidad. La norma no detalla, si esta observación directa se debe efectuar exclusivamente de forma presencial, o si podría efectuarse también a través de imágenes grabadas. Sin embargo, por ahora la Dirección del PROCET considera que el VOT no es equivalente al DOT y no avala el uso del VOT en Chile ${ }^{30}$.

La tuberculosis es una enfermedad infecciosa que amenaza la salud de la persona infectada, junto con la de toda la población. Por consiguiente, su eliminación o reducción es algo de lo que todos podemos beneficiarnos. Este interés público puede incluso prevalecer por sobre el derecho individual de aceptar o rechazar el tratamiento, y justifica el riguroso diseño de administración de medicamentos que el DOT impone sobre los pacientes en el nombre del bien común.

No obstante, en el debate ético sobre la justificación del DOT (Sagbakken 2013) ${ }^{31}$ existe una serie de condiciones necesarias para ello. Allí aparecen dos principios interesantes en el contexto del VOT. Primero está el principio del menor perjuicio (principle of least infringement), que significa, que existiendo alternativas equivalentes de intervención en el nombre del bien común, habría que buscar el método que menos vulnere la privacidad o la autonomía del paciente. Un argumento que favorece al VOT por sobre el DOT, ya que el VOT aumenta la autonomía del paciente. El segundo es el principio de la reciprocidad (principle of reciprocity), que implica que la sociedad debería compensar al paciente por la carga que se le impone en términos de tiempo y costos que debe destinar en nombre del bien común. En el caso del VOT, esto podría justificar ofrecer al paciente un smartphone con plan durante el tratamiento en caso de no poseer tal equipo.

En línea con esta idea, la OMS declara en sus "Recomendaciones sobre la ética de la prevención, atención y control de la tuberculosis" (OMS 2010) ${ }^{32}$ que: “...el respeto a la autonomía significa que los pacientes generalmente deben tener derecho a escoger entre las diferentes opciones de tratamiento..." y que "...los pacientes deben poder escoger entre diversas alternativas en cuanto a la ubicación en la que reciben el tratamiento." Esto significa, que si se comprueba que el VOT es equivalente al DOT, el paciente debería poder elegir entre ambos modos de administración.

\section{Conclusión}

En nuestro país el DOT ha sido bien establecido como norma. Los cambios en esta estrategia dependerán de las autoridades de salud. Hasta el momento esta modalidad constituye una norma vigente. Es tiempo de evaluar, si las experiencias 
positivas con VOT a nivel internacional se pueden replicar en Chile. Están dadas las condiciones tecnológicas. El VOT podría aportar a aumentar la efectividad y eficiencia del Programa TB, siempre con el objetivo de controlar y erradicar la tuberculosis.

\section{Bibliografía}

1.- BAYER R, WILKINSON D. Directly observed therapy for tuberculosis: history of an idea. Lancet 1995; 345 (8964): 1545-8.

2.- FARGA V. Hacia la erradicación de la tuberculosis. Rev Chil Enferm Respir 2006; 22: 52-4.

3.- WORLD HEALTH ORGANIZATION (WHO). Communicable Diseases Cluster. (1999). What is DOTS?: a guide to understanding the WHO-recommended TB control strategy known as DOTS.Disponible en: https:// apps.who.int/iris/handle/10665/65979.

4.- HERRERA T. Las posibles causas del aumento de la incidencia de la tuberculosis en Chile. Rev Chil Enferm Respir 2020; 36: 51-61.

5.- MINSAL. Informe de situación epidemiológica y operacional del Programa Nacional de Tuberculosis 2017. Santiago de Chile, julio de 2018, Disponible en: https://diprece.minsal.cl/wp-content/ uploads/2018/10/2018.10.05_TUBERCULOSIS-2017. pdf.

6.- MUNRO SA, LEWIN SA, SMITH HJ, ENGEL ME, FRETHEIM A, VOLMINK J. Patient adherence to tuberculosis treatment: a systematic review of qualitative research. PLoS Med 2007; 4 (7): 1230-45.

7.- DEMAIO J, SCHWARTZ L, COOLEY P, TICE A. The Application of Telemedicine Technology to a Directly Observed Therapy Program for Tuberculosis: A Pilot Project. 2001, Clin Infect Dis 2001; 15; 33 (12): 2082-4.

8.- GARFEIN RS, COLLINS K, MUNOZ F, MOSER K, CERECER-CALLU P, RAAB F, et al. Feasibility of tuberculosis treatment monitoring by video directly observed therapy: a binational pilot study. Int J Tuberc Lung Dis 2015; 19 (9): 1057-64.

9.- KUMAR AA, DE COSTA A, DAS A, SRINIVASA G, D’SOUZA G, RODRIGUES R. Mobile health for tuberculosis management in South India: is video-based directly observed treatment an acceptable alternative? JMIR Mhealth Uhealth 2019; 7 (4): e11687.

10.- NGUYEN TA, PHAM MT, NGUYEN TL, NGUYEN VN, PHAM DC, NGUYEN BH, et al. Video directly observed therapy to support adherence with treatment for tuberculosis in Vietnam: a prospective cohort study. Int J Infect Dis 2017; 65: 85-9.

11.- SEKANDI JN, BUREGYEYA E, ZALWANGO S, DOBBIN K, ATUYAMBE L, NAKKONDE D, et al. Video directly observed therapy for supporting and mo- nitoring adherence to tuberculosis treatment in Uganda: a pilot cohort study. ERJ Open Res 2020; 6 (1): 001752019.

12.- CHUCK C, ROBINSON E, MACARAIG M, ALEXANDER M, BURZYNSKI J. Enhancing management of tuberculosis treatment with video directly observed therapy in New York City. Int J Tuberc Lung Dis 2016; 20 (S): 88-93.

13.- KRUEGER K, RUBY D, COOLEY P, MONTOYA B, EXARCHOS A, DJOJONEGORO BM, et al. Videophone utilization as an alternative to directly observed therapy for tuberculosis. Int J Tuberc Lung Dis 2010; 14 (6): 779-81.

14.- MIRSAEIDI M, FARSHIDPOUR M, BANKSTRIPP D, HASHMI S, KUJOTH C, SCHRAUFNAGEL D. Video directly observed therapy for treatment of tuberculosis is patient-oriented and cost-effective. Eur Respir J 2015; 46 (3): 871-4.

15.- LAM CK, FLUEGGE K, MACARAIG M, BURZYNSKI J. Cost savings associated with video directly observed therapy for treatment of tuberculosis. Int $\mathrm{J}$ Tuberc Lung Dis 2019; 23 (11): 1149-54.

16.- WADE VA, KARNON J, ELIOTT JA, HILLER JE. Home videophones improve direct observation in tuberculosis treatment: A mixed methods evaluation. PloS One 2012; 7 (11): e50155.

17.- SINKOU H, HUREVICH H, RUSOVICH V, ZHYLEVICH L, FALZON D, DE COLOMBANI $\mathrm{P}$, et al. Video-observed treatment for tuberculosis patients in Belarus: findings from the first programmatic experience. Eur Respir J 2017; 49 (3): pii: 1602049. Doi:10.1183/13993003.02049-2016.

18.- MACARAIG M, LOBATO MN, MCGINNIS PILOTE K, WEGENER D. A National Survey on the Use of Electronic Directly Observed Therapy for Treatment of Tuberculosis. J Public Health Manag Pract 2018; 24 (6): 567-70.

19.- RITTER LA, KWONG MW, NASSERI LM. California Public Health Departments Remotely Treat Tuberculosis: Outcomes \& Opportunities. Californian Journal of Health Promotion 2017; 15 (2): 37-45.

20.- GARFEIN RS, RIDDHI P. Doshi. Synchronus and synchronus video observed therapy (VOT) for tuberculosis treatment adherence monitoring and support. J Clin Tuberc Other Mycobact Dis. Disponible en: https://dol. org/10.1016/j.jctube.2019. 100098.

21.- WHO. Digital Health for the End TB Strategy. An agenda for action. 2015. Disponible en: http://www.who.int/ tb/publications/digitalhealth-TB-agenda/en/).

22.- WHO. Global Task Force on digital health for TB.Disponible en: www.who.int/tb/areas-of-work/ digital-health/global-task-force/en/ accedido: 20.05 . 2020

23.- FALZON D, TIMIMI H, KUROSINSKI P, MIGLIORI G, VAN GEMERT W, DENKINGER C, et al. Digital health for the End TB Strategy: developing 
priority products and making them work. Eur Respir J 2016; 48: 29-45.

24.- WHO. Guidelines for treatment of drug-susceptible tuberculosis and patient care, 2017. Page 16: Disponible en: https://apps.who.int/iris/bitstream/hand le/10665/255052/9789241550000-eng.pdf?sequence $=1$. Accedido 20.5.2020.

25.- RAVENSCROFT L, KETTLE S, PERSIAN R, RUDA S, SEVERIN L, DOLTU S, et al. Video observed therapy (VOT) and medication adherence for TB patients: RCT in Moldova. Eur Respir J 2020; 2000493. Doi:10.1183/13993003.00493-2020.

26.- STORY A, ALDRIDGE RW, SMITH CM, GARBER E, HALL J, et al. Smartphone-enabled video observed versus directly observed treatment for tuberculosis: a multicentre, analyst blinded, randomised, controlled superiority trial. Lancet 2019; 393 (10177): 1216-24.

27.- NHS England, Commission use of the Find and Treat Video Observed Therapy on the SureAdhere platform to support tuberculosis treatment completion. 26/2/2020. Disponible en: https://www.england.nhs.uk/Itphemenu/ better-care-tuberculosis/find-and-treat-video-observedtherapy.
28.- SUBTEL. Subsecretaría de Telecomunicaciones. Gobierno de Chile. IX Encuesta de Acceso y Usos de Internet. 2017. Disponible en: https://www.subtel.gob. cl/wp-content/uploads/2018/07/Informe_Final_IX_Encuesta_Acceso_y_Usos_Internet_2017.pdf.

29.- MINISTERIO DE SALUD. Gobierno de Chile. Normas técnicas para el control y la eliminación de la tuberculosis, MINSAL 2014, p 28. Art. 95.

30.- Carta de Dra. Nadia Escobar, Directora Programa de Control y Eliminación de la Tuberculosis (PROCET), 16.06.2020.

31.- SAGBAKKEN M, FRICH JC, BJUNE GA, PORTER JD. Ethical aspects of directly observed treatment for tuberculosis: a cross-cultural comparison. BMC Med Ethics 2013; 14: 25. 10.1186/1472-6939-14-25.

32.- WHO. Guidance on ethics of tuberculosis prevention, care and control. 2010 version en español: Recomendaciones sobre la ética de la prevención, atención y control de la tuberculosis Organización Panamericana de la Salud, Washington 2013. Revisado 03.06.2020. citas paginas 7 y 13 Disponible en: https://apps.who.int/ iris/bitstream/handle/10665/89637/9789275317433_spa. pdf? sequence $=1$.

Correspondencia a:

Dr. Robert Hartog, MPH

CESFAM Cristo Vive. Recoleta. Santiago, Chile.

Email: rhartog@fundacioncristovive.cl 